\section{Predictors of Depression, Anxiety, Anticipatory Grief and Growth in Turkish Offspring as} Caregivers of Parents with Alzheimer's disease.

Yagmur Ar-Karci, TED University

A. Nuray Karanci, Middle East Technical University

Introduction: Unpaid AD caregivers may

become hidden patients who are vulnerable to a variety of physical and psychological ill health effects due to caregiving difficulties 1 .

This unpaid caregiving trend is much more common in under-developed or developing countries because of the financial restrictions and societal views on care provision 1,2 . Turkey is also a developing country, dominantly influenced by Islamic values and collectivist norms. Informal caregiving of $A D$ patients has become exponentially common over time as a result of the increasing incidence of $A D$ and the cultural views encouraging familial care provision and the scarcity of alternative care institutions 3,4.

Particularly, although well-established relations were proposed between disease related variables and caregiver outcomes, more research seems to be needed to explore the impacts of modifiable caregiver related factors in $A D$ caregiving both in the world and in Turkey5,6,7. Hence, the main aim of this study was to understand Turkish adult children caregivers' experiences of caring for a parent with AD. Accordingly, the research questions were formed as follows:

1. Does coping strategies moderate the relationship between caregiver burden and caregiving outcomes?

2. Does perceived social support moderate the relationship between caregiver burden and caregiving outcomes?

H CH, Sanders S, Kelber ST. 2007. Grief and personal growt experience of spouses and adult-child caregivers of individuals with Alzheimer's disease and related dementias.

Peacock, S., Forbes, D., Markle-reid, M., Hawranik, P., Morgan D., Jansen, L., ... Henderson, S. R. (2010). The Positive Aspect of the Caregiving Journey With Dementia: Using a Strength Bsed Perspective to Reveal Opportunities Lopez, J., Lopez-Arrieta, J., \& Crespo, M. (2005). Factors associated with the positive impact of caring for elderly and dependent relatives.

\section{Participants}

$\checkmark 190$ Turkish adult children caring for a parent with AD.

$\checkmark$ The eligibility criteria for participation were (1) being an adult child of a patient with AD, (2) defining oneself as an informal caregiver, (3) giving some assistance in daily and instrumental living activities and (4) providing at least four hours of caregiving per week.

The mean age of the participants was 51.41 $(S D=8.68)$, ranging from 26 to 77 .

$\checkmark$ Eighty-nine percent of the sample were female $(N=170)$, while $11 \%$ was male $(N=$ 20).

More than half of the caregivers had education beyond high school ( $N=109,54.7 \%)$. Almost half of the caregivers reported their income level as equal or more than $2500 \mathrm{TL}(N=85$, $44.7 \%)$

Caregiving to mother $(N=144, \% 76)$,

Caregiving per week $(M=103.57, S D=65.92)$, Length of Caregiving ( 5.23 years on average, ( $S D=3.46)$, Co-Residence $(N=129,67.9 \%)$

\section{Instruments}

$\checkmark$ Demographic Information Form

$\checkmark$ Beck Depression Inventory

$\checkmark$ STAI-State Form

$\checkmark$ Marwit-Meuser Caregiver Grief Inventory-Shor Form

$\checkmark$ Post Traumatic Growth Inventory

$\checkmark$ Zarit Burden Inventory

$\checkmark$ Ways of Coping Inventory (WCl)

$\checkmark$ Multidimensional Scale of Perceived Social Support (MSPSS)

Statistical Analysis: The moderator roles of coping strategies (i.e. fatalistic coping, optimistic/seeking social support, problem-solving coping and helplessness coping) and perceived social support on caregiver outcomes (i.e. depression, anxiety, grief and growth) were investigated through conducting a set of moderation analyses. Hayes and Matthes (2009) macro was used in order to perform moderation analyses

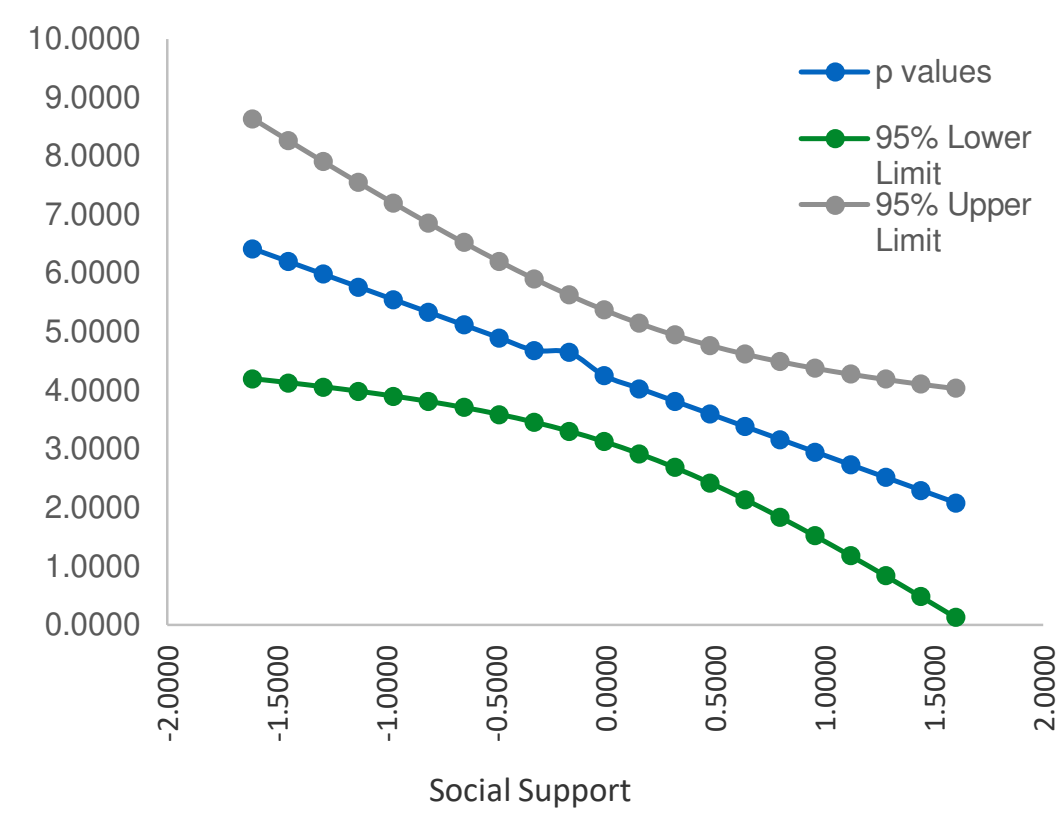

Figure 2. Relationship between Caregiver Burden and Depression for Different Scores of Perceived Social Support with the Confidence Interval Values

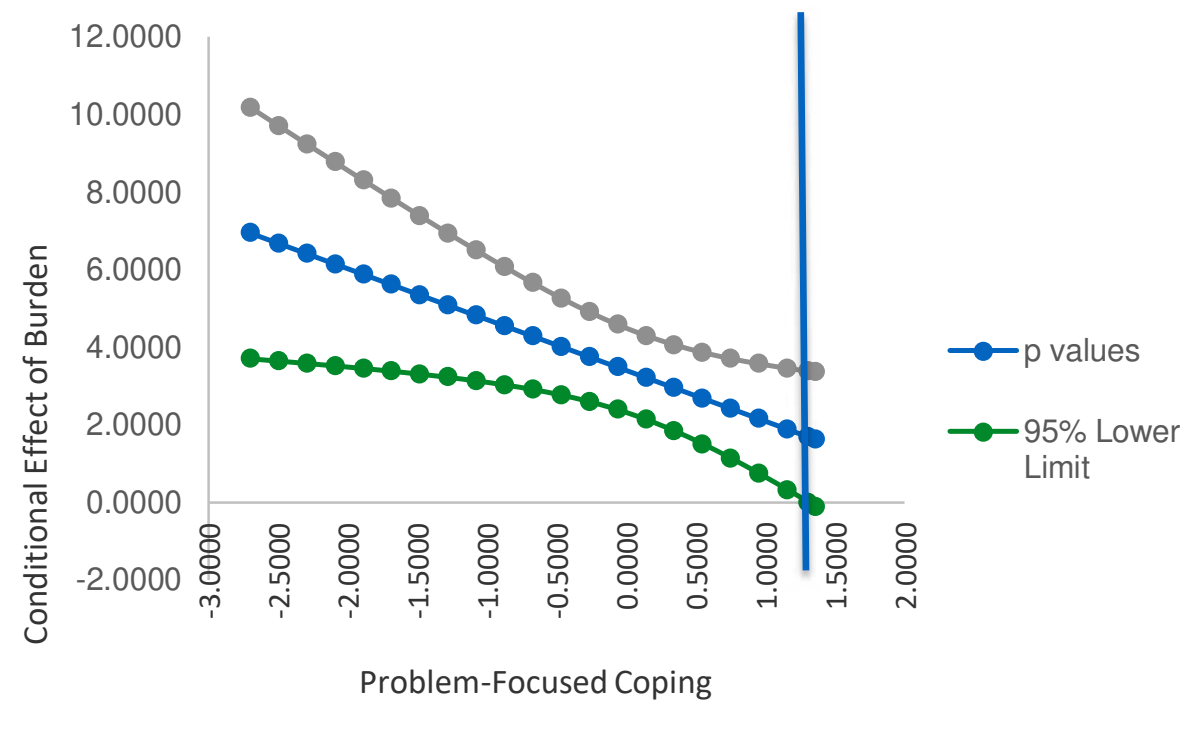

Figure 2. Relationship between Caregiver Burden and Depression for Different Scores of Problem Focused Coping with the Confidence Interval Values

References

Adams, B. K. \& Sanders, S. (2004). Alzheimer's caregiver differences in experience of loss, grie reactions and depressive symptoms across stage of disease.

Ar, Y. \& Karanci, N. Turkish adult children as caregivers of parents with Alzheimer's disease: Perceptions and caregiving experiences. Hilgeman MM, Durkin DW, Sun F, et al. (2009). Testing a theoretical model of the stress process in Alzheimer's caregivers with race as a moderator. Jeongim, H. G. (2014). Religious Coping, Positive Aspects of Caregiving, and Social Support Among Alzheimer's Disease Caregivers.

\title{
Discussion
}

$\checkmark$ Caregiver depression tended to decrease at higher values of caregiver burden. By contrast, when the perceived social support level was lower, caregiver depression tended to increase at higher values of caregiver burden.

$\checkmark$ When caregivers used problem-solving coping strategies less, caregiver depression tended to increase at higher levels of caregiver burden. Nevertheless, higher levels of problem-focused coping did not have an effect on the relationship between caregiver burden and depression

$\checkmark$ When the levels of perceived social support decreased, growth levels of caregivers tended to decrease at the higher values of caregiver burden. By contrast, when the levels of perceived social support increased, caregivers experience greater growth even at the higher levels of caregiver burden

$\checkmark$ Importance of culturally sensitive multi-component intervention programs

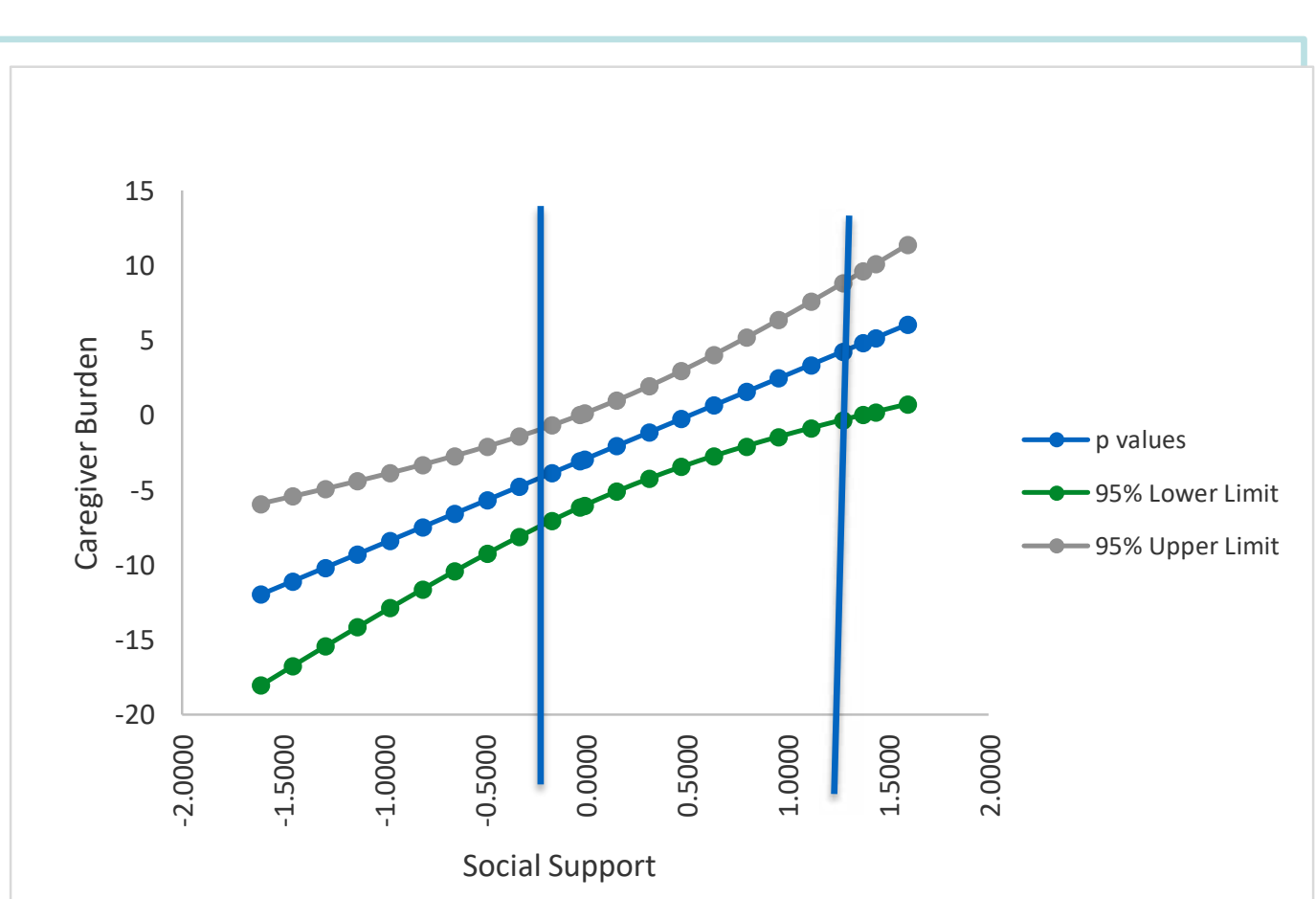

Figure 3. Relationship between Caregiver Burden and Growth for Different Scores of Perceived Social Support with the eropfidences Interval Values 